\title{
Flavonoid Biosynthesis Pathway Participating in Salt Resistance in a Landrace Sweet Sorghum Revealed by RNA-Sequencing Comparison With Grain Sorghum
}

\author{
Ren Genzeng ${ }^{1}$, Cui Jianghui ${ }^{1}$, Xiang Xiaodong ${ }^{1}$, Gao Yukun ${ }^{1}$, Wei Shilin ${ }^{1}$, Chang Jinhua ${ }^{1} \&$ Xu Hao ${ }^{2}$ \\ ${ }^{1}$ North China Key Laboratory for Crop Germplasm Resources of Education Ministry, College of Agronomy, \\ Hebei Agricultural University, Baoding, Hebei, China \\ ${ }^{2}$ College of Resources and Environmental Science, Hebei Agricultural University, Baoding, Hebei, China \\ Correspondence: Chang Jinhua, North China Key Laboratory for Crop Germplasm Resources of Education \\ Ministry, College of Agronomy, Hebei Agricultural University, Baoding, Hebei, China. E-mail: \\ jhchang2006@126.com \\ Xu Hao, College of Resources and Environmental Science, Hebei Agricultural University, Baoding, Hebei, \\ China.E-mail: xuhao22003@126.com
}

Received: January 28, 2019

Accepted: March 9, 2019 Online Published: May 15, 2019

doi:10.5539/jas.v11n6p63

URL: https://doi.org/10.5539/jas.v11n6p63

\begin{abstract}
Abiotic stresses affect crop productivity worldwide. Plants have developed defense mechanisms against environmental stresses by altering the gene expression pattern which leads to regulation of certain metabolic and defensive pathways. Sorghum [Sorghum bicolor (L.) Moench] is an important crop in those regions irrigated by salty water. Sweet sorghum is a variant of common grain sorghum and is relatively more adapted to marginal growing conditions. Here, we compared the different response to salt stress of sweet and grain sorghum. We investigated six traits related with seed germination under salt-stress and normal conditions, conducted a genome-wide research on the salt effect on the gene expression of a landrace sweet and two grain sorghum by RNA-sequencing at seedling stage. The results showed that salt stress had significant inhibition to sorghum seed germination capability, and the inhibition to grain sorghum was greater. By comparing sweet and grain sorghum and the KEGG pathway analysis based on the DEGs, six genes involved in flavonoid biosynthesis pathway to tannins and anthocyanins from phenylalanine were identified in the landrace sweet sorghum, which expression was significant different with that in grain sorghum. Quantitative real-time PCR (qRT-PCR) data were closely in accordance with the transcript patterns estimated from the RNA-seq data. Tannins accumulation changes were associated with the genes expression under salt stress and control. These suggested that flavonoid biosynthesis pathway was involved in the sorghum resistance to salt stress. The present results suggested that flavonoid biosynthesis plays an important role in the sweet sorghum capacity for salt tolerance.
\end{abstract}

Keywords: Sorghum, salt stress, flavonoid biosynthesis, tannins, RNA-sequencing

\section{Introduction}

Salt stress is one of the main restrictions to crop production as it affects more than 800 million hectares (ha) of land worldwide (Farooq et al., 2017). Moreover, salinity stress is a major environmental threat to agriculture and its adverse impacts are getting more serious in those salt affected areas (Johnson et al., 1992; Zeng et al., 2001). Salinity stress involves a complex process such as osmotic stress, ionic imbalances, and sets in secondary stresses such as accumulation of toxins, nutritional imbalances and oxidative stress in plants, ultimately causing a reduction in plant growth and development (Munns \& Tester, 2008; Türkan \& Demiral, 2009). Defense strategies of plants against salinized conditions depend upon activation of cascades of molecular networks involved in stress sensing, signal transduction and the expression of specific stress-related genes and metabolites (Türkan \& Demiral 2009; Deinlein et al., 2014; Wang et al., 2010). Understanding the molecular mechanisms of abiotic stress tolerance is therefore critical for developing stress tolerant crop plants and use of saline water in the future.

Sorghum (Sorghum bicolor L. Moench) is an important crop widely grown in the arid and semi-arid regions. Sweet sorghum is a variant of common grain sorghum and a widely adapted sugar crop with potential for 
bioenergy production (Carpita \& McCann 2008; Rooney et al., 2007; Vermerris, 2011; Regassaand \& Wortmann 2014). It is relatively more adapted to marginal growing conditions, such as salinity, alkalinity, water stress and other environmental stresses compared with grain sorghum and maize (Zhang et al., 2011; Michael et al., 2017; Regassa \& Wortmann 2014). Its remarkable levels of salinity tolerance make it a good candidate to identify salt-resistance genes.

Many genes expression is induced or suppressed by salt stress, and the study on gene expression regulation has become a hot topic in recent years. The release of genome sequence of sorghum and next/third generation sequencing (NGS) technologies provide a favorable reference to investigate interaction mechanisms between environment stress and sorghum genes response (Paterson et al., 2009; Klára Kosová et al., 2011; Liu et al., 2012; Martin et al., 2011; Suja et al., 2017). This study, therefore, compared sweet and grain sorghum seedlings responses to salt stress based on RNA-sequencing and found genes and pathways in sweet sorghum which may participate in salt stress response by regulation of flavonoid biosynthesis. Our results presented here help us elucidate the salinity stress effects and develop salinity management strategies aimed at enhancing the use of saline water in agriculture based on plant improvements.

\section{Material and Methods}

\subsection{Evaluation of Seed Germination Under Salt Stress}

Three sorghum varieties were selected as materials; i.e., a landrace sweet sorghum variety, GZ, which has high tolerance to salt; two grain sorghum cultivars, TAx623B (genome sequenced) and HN16, which are relative susceptible to salt. Before germination experiment, the seeds were surface-sterilized using $15 \%$ bleach for 20 min and washed subsequently three times with sterile distilled water. 30 seeds from each cultivar were placed in Petri dishes on two layers of filter paper soaked with $10 \mathrm{~mL} \mathrm{NaCl}$ solution. The filter paper was soaked with 10 $\mathrm{mL}$ distilled water in the control. Then the seeds were cultured in an illumination incubator at day/night temperature $30{ }^{\circ} \mathrm{C} / 25^{\circ} \mathrm{C}$ with 10 -h light/14-h dark photoperiod under relative humidity of $80 \%$ for 10 days. Four different $\mathrm{NaCl}$ concentrations (40 (S1), 80 (S2), 120 (S3) and $160 \mathrm{mM}$ (S4)) were set in order to compare the sorghum different response to stress and determine the most suitable salt concentration for further experiment. Each treatment was performed three biological replicates. Distilled water was used to supplement the daily evaporation to maintain $\mathrm{NaCl}$ concentration. Seeds germination index (GI), vigor index (VI), germination rate potential (GP), germination rate (GR) and inhibition rate (IR) were calculated.

The GR represented the germination percentage at 7 days $(\mathrm{GR}=\mathrm{Nt} / \mathrm{N} 0 \times 100 \%$, $\mathrm{Nt}$ is the number of germinated seeds at day 7 and N0 is the total number of experimental seeds). GP was the germination percentage at 4 days after treatment. GI was calculated by the formula: $\mathrm{GI}=\Sigma(\mathrm{Gt} / \mathrm{Tt})(\mathrm{Gt}$ is the accumulated number of germinated seeds at day $\mathrm{t}$ and $\mathrm{Tt}$ is the time corresponding to $\mathrm{Gt}$ in $\mathrm{t}$ days). 10 germinated seeds were chosen to measure the average root length (RL)), root dry weight (RDW) and leaf dry weight (LDW) after 10 days. The seed vigor index (VI) was measured by the formula: $\mathrm{VI}=\mathrm{S} \times \mathrm{GI}$ ( $\mathrm{S}$ is the accumulated fresh root weight). The response to salt stress was evaluated with inhibition ratio (IR): IR $=(1-\mathrm{Ps} / \mathrm{Pc})$, (Ps is mean performance of a cultivar under stress; Pc represents average performance of the same cultivar under control) (Wang et al., 2010).

\subsection{Plant Culture and RNA Isolation}

For salt stress treatments, three-leaves-old sorghum plants were irrigated with $160 \mathrm{mM} \mathrm{NaCl}$. Whole plants were collected at 0,48 and $72 \mathrm{~h}$ after treatment and stored at $-80{ }^{\circ} \mathrm{C}$ for mRNA isolation (the treatment code was as shown in Table 1). Each collection was replicated three times (every sample consisted of 5 plants). Total sorghum RNA was extracted using Trizol plus RNA kit according to the manufacturer's instructions. The RNA from three replicates was mixed at 1:1:1 ratio. RNA quality and integrity were measured using Nanodrop TM 1000 Spectrophotometer (Thermo, MA, USA). Qualified RNA was used for subsequent RNA library construction and sequencing. 
Table 1 . The codes of samples treated by $\mathrm{NaCl}$

\begin{tabular}{ll}
\hline Samples & Code \\
\hline TA $\times 623$ B-ck $(0$ h stress $)$ & T01 \\
GZ-ck $(0$ h stress $)$ & T02 \\
HN16-ck $(0$ h stress $)$ & T03 \\
TA $\times 623 B-48$ h stress & T04 \\
GZ-48 h stress & T05 \\
HN16-48 h stress & T06 \\
TA $\times 623 B-72 ~ h$ stress & T07 \\
GZ-72 h stress & T08 \\
HN16-72 h stress & T09 \\
\hline
\end{tabular}

\subsection{Preparation of RNA Library and Sequencing}

Poly (A) mRNAs were purified from total RNA using oligo (dT) magnetic beads and cut into short fragments with fragmentation buffer. These fragments were used as templates for first-strand cDNA synthesis using random hexamer primers. The second strand cDNA was synthesized using Buffer, dNTPs, DNA polymerase I and RNase H. Double-stranded cDNA was purified by AMPure XP beads, and then end-repaired. Sequencing adaptors were ligated to the fragments and the desired size fragments were selected to be enriched by PCR amplification (Wang, et al., 2017). The amplified products were sequenced using an Illumina HiSeq 2500 platform (Illumina, San Diego, CA, USA.).

\subsection{Identification of Differentially Expressed Genes (DEGs) and Gene Annotation}

Raw data were quality-filtered with criteria of less than $0.1 \%$ low quality bases (Phred score $<30$ ). Adapter sequences and primers were subsequently removed. The filtered reads were aligned to the sorghum reference genome using TopHat2 software (Love et al., 2014). The sequences were further annotated by GO, KOG, Swiss-Prot, KEEG, COG and NR databases with criteria of E-value equals $10^{-5}$. The GO terms and KEGG pathway analysis were conducted using Blast2Go. Fragments per kilo base of exon per million fragments mapped (FPKM) and DESeq (Anders \& Huber, 2010) were employed to represent gene expression level. DEGs were measured with Fold Change $\geq 2$ and FDR $<0.01$.

FPKM were estimated by following equation:

$$
\text { FPKM }=\frac{\text { cDNA Fragments }}{\text { Mapped Fragments (Millions) } \times \text { Transcript Length }(\mathrm{kb})}
$$

\subsection{Gene Expression Analysis by $q R T-P C R$}

To evaluate the genes associated with salt stress response mined from the Illumina data, transcriptomes obtained from the comparison between sweet and grain sorghum were selected to validate the RNA-seq results by qRT-PCR with Actin (LOC110436378) as the endogenous control. Gene-specific primer sequences were designed using Primer 3 software and are listed in Table 2. RNA for qRT-PCR analysis was extracted from 0,48 and $72 \mathrm{~h} \mathrm{NaCl}$ stressed plants (RNA and cDNA were as same as RNA sequencing). Non-template controls were performed in every reaction. Relative transcription levels were calculated using the $2^{-\Delta \Delta C t}$ method (Livak \& Schmittgen, 2001). qRT-PCR data shown were averages of three technical replicates for each independent experiment. 
Table 2. Primer for qRT-PCR

\begin{tabular}{|c|c|c|}
\hline Sobic.001G249600F & & cct cct gga cct ggc act cg \\
\hline Sobic.001G249600R & & cct cgt gaa ctg cac cac ctt g \\
\hline Sobic.003G230900F & & tcg get cet ggc tcg tca tg \\
\hline Sobic.003G230900R & & cag caa tgg ctt cgt ctt cac aac \\
\hline sobic.006G272700F & & gct gcc gcc tta tac tgt cat ctg \\
\hline sobic.006G272700R & & gac get tgt tcc ttc tcg gtg ac \\
\hline sobic.006G253900F & & gcg gtg gtg aac tct gag tgt ag \\
\hline sobic.006G253900R & & cca tct tgc ggc ggt aca tct $c$ \\
\hline sobic.006G227000F & & cac cgt gaa gac aca tct gct ctc \\
\hline Sobic.006G227000R & & cgt cca gcg tct cgt act tga ac \\
\hline sobic.004G000700F & & cca cta cge tge ttg cca tcc \\
\hline Sobic.004G000700R & & $\operatorname{tgc} \operatorname{tg} c \operatorname{tgc} \operatorname{tg} c \operatorname{tgc} \operatorname{tg} t \operatorname{tc}$ \\
\hline \multirow[t]{2}{*}{ actin } & $\mathrm{F}$ & ggtcctcttccagccatcctt \\
\hline & $\mathrm{R}$ & atttccttgcctcatcctgtca \\
\hline
\end{tabular}

\subsection{Determination of Tannins Content}

The seedlings were washed with fresh water, chopped into small pieces and oven-dried (samples were collected at the same time with RNA sequencing). The material was ground to fine powder using an electric grinder. Tannins were determined by the acid butanol assay (Terrill et al., 2010). Tannic acid was also used in this study as standard. Every sample was performed three biological replicates.

Total tannins content was expressed as milligrams tannic acid equivalents per gram of dry plant extract $(\mathrm{mg} / \mathrm{g})$ through the calibration curve of tannic acid, whose linearity range was from 10 to $100 \mathrm{mg} / \mathrm{mL}\left(\mathrm{R}^{2}>0.99\right)$.

\subsection{Data Analysis}

Data were analyzed using EXCEL 2003 (Microsoft Corporation, WA, USA) and SPSS 17.0 (IBM SPSS, NY, USA.).

\section{Result}

\subsection{Salt Stress Effects on Seed Germination of Sweet and Grain Sorghum}

GI, VI, GR, GP and IR were calculated for 3 sorghum cultivars. The seed germination capability significantly decreased as $\mathrm{NaCl}$ concentration increased. The inhibition of salt stress on GR, VI, RDW and RL was significantly different between the sweet and grain sorghum $(\mathrm{p}<5 \%$, Table 3 , Figure 1$)$. The inhibition of salt stress to the sweet sorghum seedlings was the least, which showed GZ has the best tolerance to salt (Figure 2). The most inhibition was observed under $160 \mathrm{mM} \mathrm{NaCl}$ treatment. Therefore, $160 \mathrm{mM} \mathrm{NaCl}$ treatment was used to compare the different responses to salt stress between the sweet and grain sorghum. Whole plants were collected at $48 \mathrm{~h}$ and $72 \mathrm{~h}$ salt stress and control for RNA-sequencing.

Table 3. The inhibition of salt stress on sorghum seed germination (IR)

\begin{tabular}{llllllll}
\hline Cultivar & GR & GP & GI & RDW & LDW & VI & RL \\
\hline GZ & $0.0904 \mathrm{~b}$ & $0.361 \mathrm{~b}$ & $0.2127 \mathrm{a}$ & $0.5366 \mathrm{~b}$ & $0.4585 \mathrm{~b}$ & $0.576 \mathrm{~b}$ & $0.5182 \mathrm{~b}$ \\
BTx623B & $0.1335 \mathrm{a}$ & $0.3571 \mathrm{~b}$ & $0.2598 \mathrm{a}$ & $0.6068 \mathrm{a}$ & $0.4275 \mathrm{~b}$ & $0.6381 \mathrm{a}$ & $0.6857 \mathrm{a}$ \\
HN16 & $0.1682 \mathrm{a}$ & $0.6214 \mathrm{a}$ & $0.2791 \mathrm{a}$ & $0.6030 \mathrm{a}$ & $0.6465 \mathrm{a}$ & $0.6587 \mathrm{a}$ & $0.7252 \mathrm{a}$ \\
\hline
\end{tabular}

Note. Different letters in the same column indicate significant difference among treatments at $\mathrm{p}<5 \%$. 


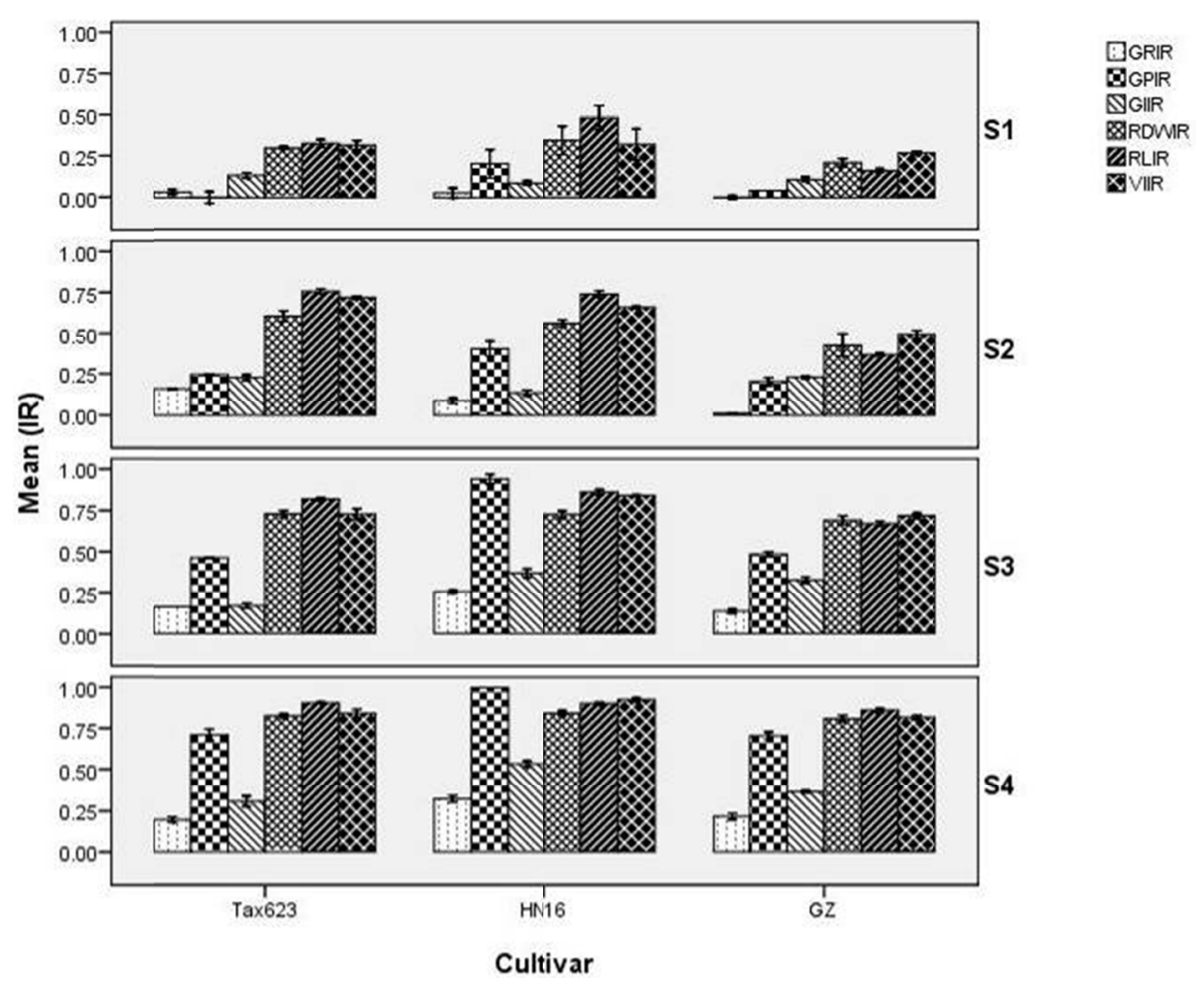

Figure 2. The responses of the genotypes to salt stress (IR) at different salt concentration. Error bars represent standard errors from three biological replicates 


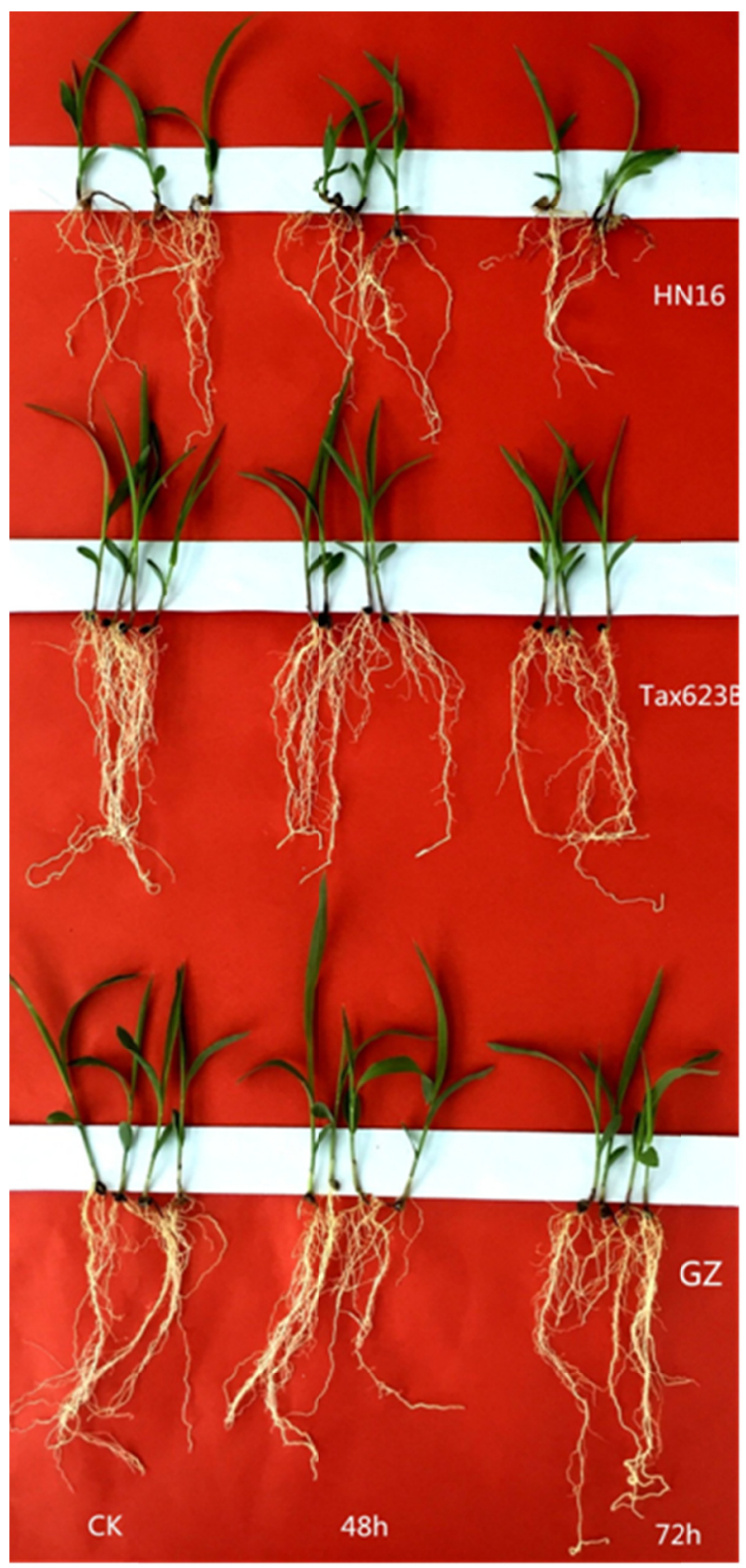

Figure 3. The different responses to salt stress at different stress time of sorghum seedings

\subsection{NaCl-Stress Effect on Gene Expression in Sweet and Grain Sorghum}

Different $\mathrm{NaCl}$-stress effect on gene expression in sweet and grain sorghum was compared by RNA-sequecing. After filtering raw reads, the clean reads resulted in 66361 transcripts with more than 1000bp length (Supplementary Table 1, Note 1) out of all reads. With comparison by DEGSeq20 software analysis $(\mathrm{p} \leq 0.01$ and fold change $\geq 2$ ), 561 up and 1092 down regulated genes in GZ compared with two grain sorghum under control (Supplementary Tables 2 and 3) were identified. By further comparing the genes expression at different salt-treatment time in every variety and among different varieties, out of the 561 DEGs, 8 genes were observed overlapping up-regulated DEGs and 26 out of 1092 down-regulated genes were identified overlapping genes among 3 sorghum cultivars either in control or in $48 \mathrm{~h}$ salt treatment (Figures $3 \mathrm{~A}$ and 3B; Supplementary Tables 4 and 5). Their expression increased at $48 \mathrm{~h}$ salt stress and then decreased or had no significant changes at $72 \mathrm{~h}$ in every sorghum variety. 5 genes (Sobic.003G230900, Sobic.004G000700, Sobic.006G227000, Sobic.006G253900, Sobic.008G026200) out of the 8 DEGs expressed higher in GZ than in grain sorghum 
overtime salt stress (from $0 \mathrm{~h}$ to $72 \mathrm{~h}$ ). Out of the 26 down-regulated genes, 8 genes were expressed lower in GZ than in two other sorghum varietie from $0 \mathrm{~h}$ to $72 \mathrm{~h}$ salt stress (Supplementary Table 5).

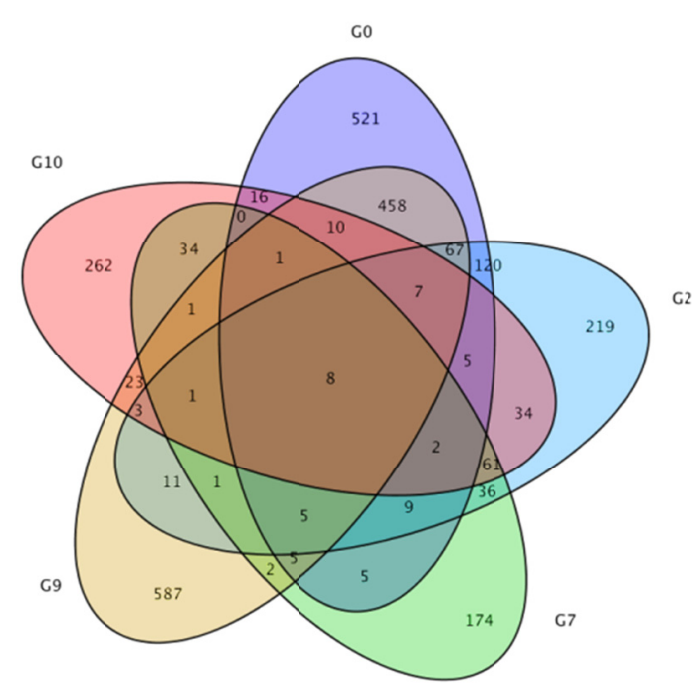

A

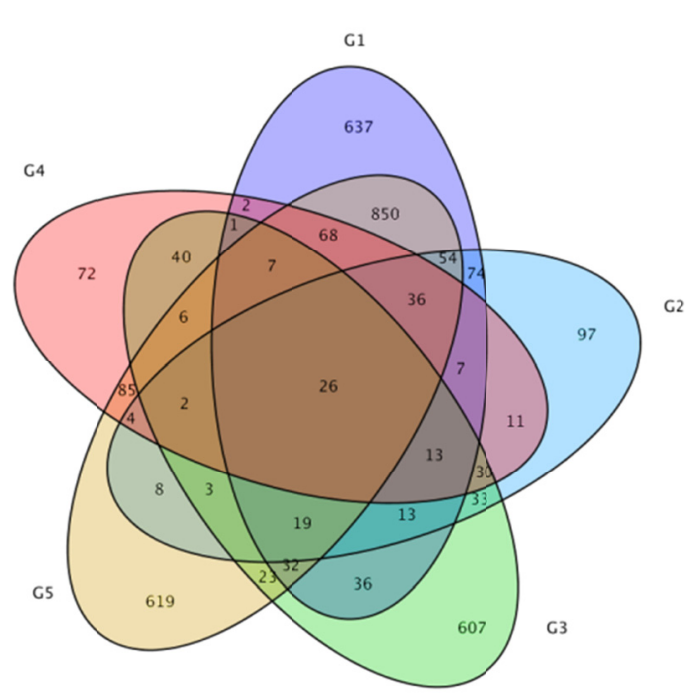

B

Figure 3. Venn diagram of up- and down-regulated genes at different time-points between sweet and grain sorghum after salt treatment. A, Common up regulated genes in 3 sorghum cultivars under $48 \mathrm{~h} \mathrm{NaCl}$ stress and control. B, Common down regulated genes in 3 sorghum cultivars under $48 \mathrm{~h} \mathrm{NaCl}$ stress and control

Note. G1:T01 vs T02; G2:T01 vs T04; G3:T02 vs T05; G4:T03 vs T06; G5:T03 vs T02.

\subsection{Enriched Functions and Pathways of the DEGs Between the Sweet and Grain Sorghum}

We developed a keen interest in the overlapping genes which expressed different overtime under salt treatment. To further explore the major biological functions of the DEGs, pathway enrichment analyses were conducted, based on GO enrichment, COG, KOG and KEGG analysis, using a threshold p-value $\leq 0.05$. The GO enrichment analysis indicated that the 8 genes were involved into binding and catalytic activity under the molecular process (MP) category. The 8 genes were mainly located in the cell, cell part and organelle, and the cellular component (CC) category; and in the biological process (BP) category, the 8 genes were mostly involved in response to stimulus (Figure 4). These results indicated that these genes played an important role in the response to stress in GZ, particularly Sobic.003G230900 and Sobic.006G227000 which were found to be involved in defense mechanisms. Based on KEGG database, 4 genes (Sobic.003G230900, Sobic.004G000700, Sobic.006G227000, Sobic.006G253900) out of the 5 up-regulated DEGs overtime in GZ comparing to grain sorghum were allocated in flavonoid biosynthesis pathway (ko00941) (Figure 5), which are responsible for Dihydroflavonol-4-reductase (DFR), Leucoanthocyanidin dioxygenase (LDOX), Anthocyanidin reductase (ANR) and Naringenin,2-oxoglutarate 3-dioxygenase (F3H) enzymes. Sobic.003G230900 was related with the response to salt stress by GO annotation. Sobic.006G227000 and Sobic.003G230900 were included in defense mechanisms based on KOG class annotation (Supplementary Table 6). Meanwhile, 2 genes (Sobic.001G444400, Sobic.004G340200) out of the 8 overtime down-regulated DEGs code peroxidase (POD) and cinnamoyl-CoA reductase (CCR) which lead phenylalanine to biosynthesis of lignin in phenylpropanoid biosynthesis (ko00940) annotated by KEGG classification (Table 4). These results indicated these genes participate in flavonoid biosynthesis pathway and contribute to flavonoids accumulation. 


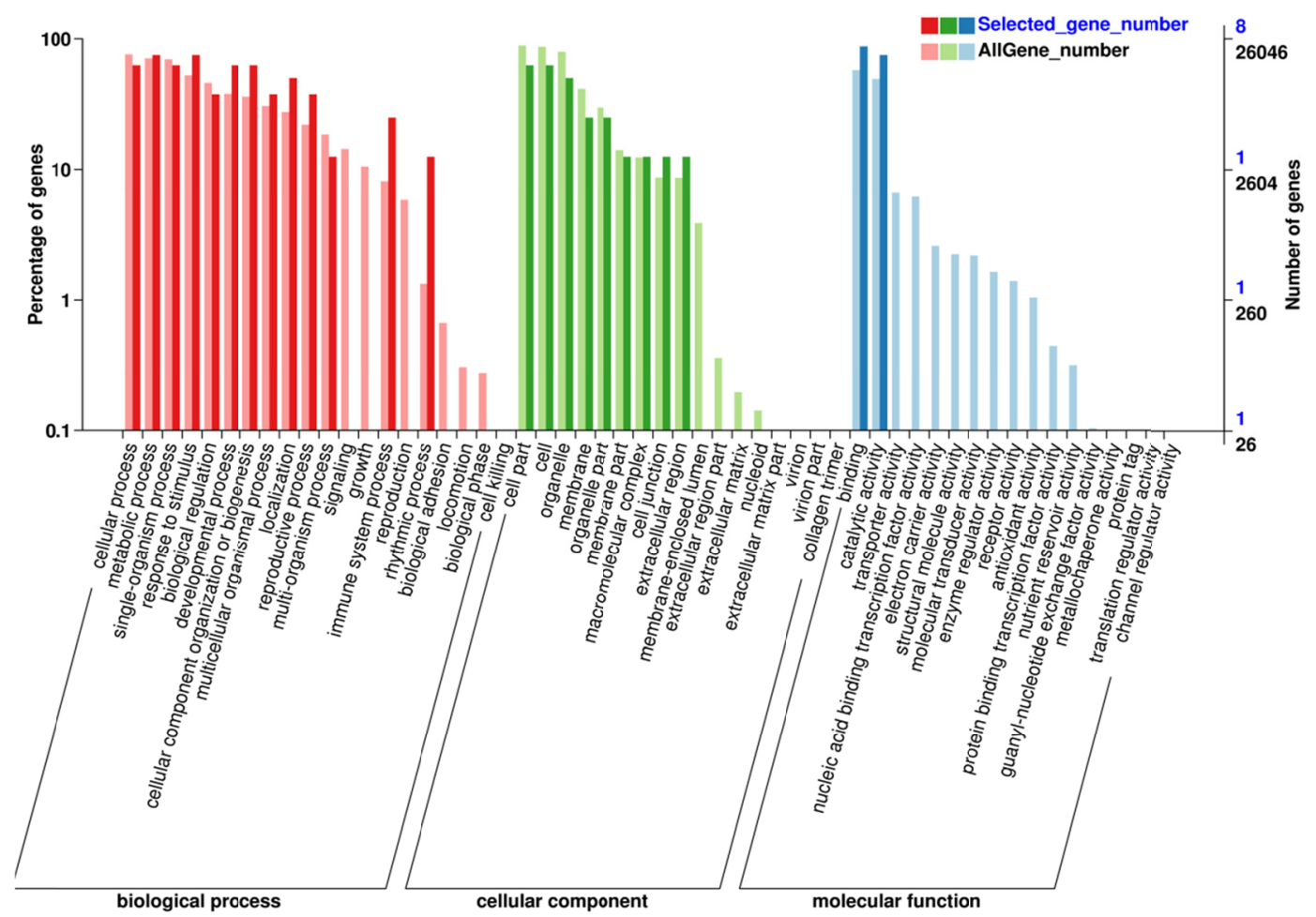

Figure 4. GO annotation of the eight up regulated genes under control and $48 \mathrm{~h}$ salt stress in three sorghum cultivars

FLAVONOID BIOSYNTHESIS

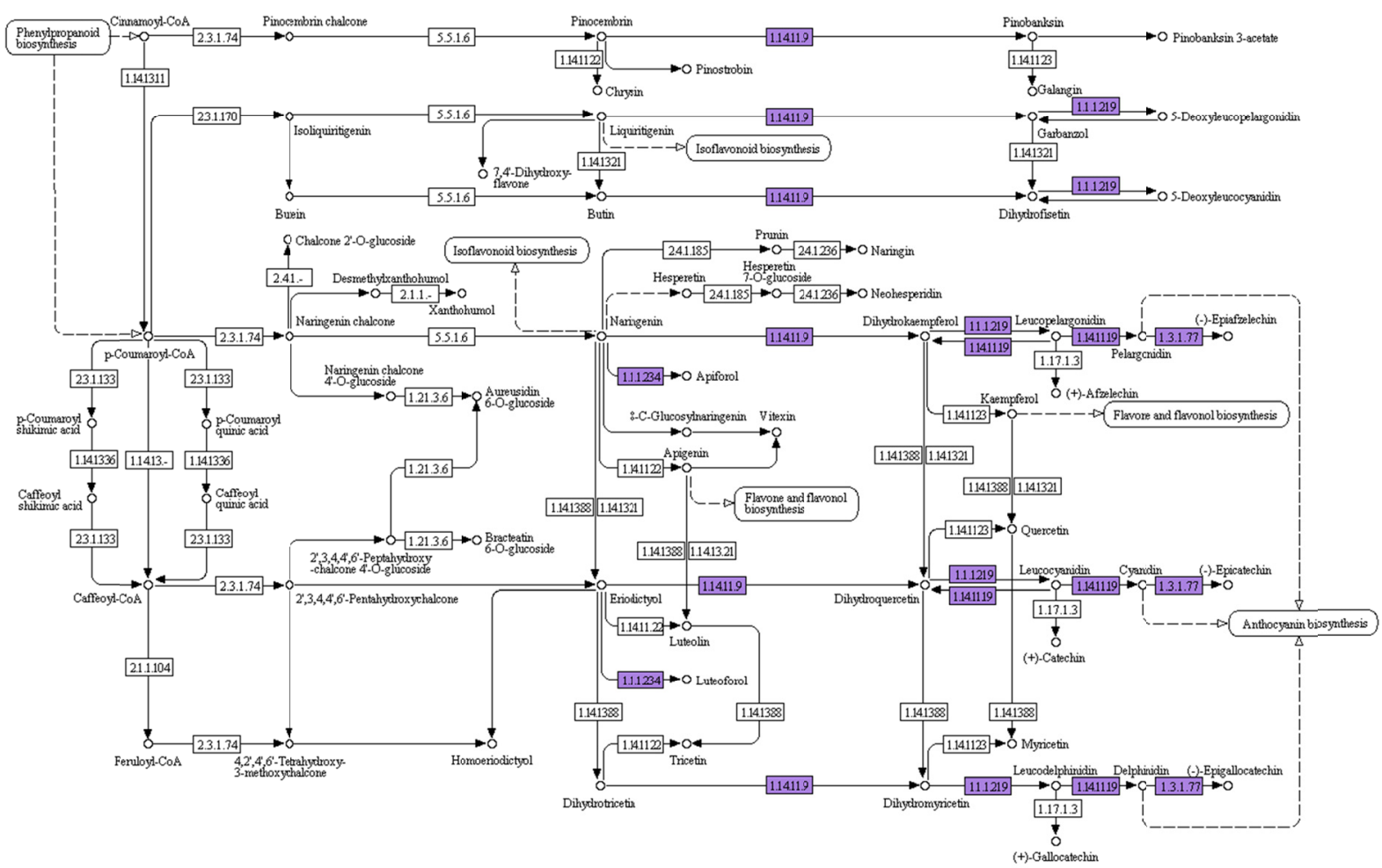

Figure 5. KEGG pathway of 8 up regulated DEGs at $48 \mathrm{~h}$ salt stress in three sorghum cultivars 
Table 4. Annotation of 8 up-regulated and 2 down -regulated genes

\begin{tabular}{lllll}
\hline Gene ID & Chro-some & Regulation & Function (enzymes) & KEGG Classfication \\
\hline Sobic.003G230900 & 3 & $\mathrm{Up}$ & DFR & Flavonoid biosynthesis (ko00941) \\
Sobic.004G000700 & 4 & $\mathrm{Up}$ & LDOX & Flavonoid biosynthesis (ko00941) \\
Sobic.006G227000 & 6 & $\mathrm{Up}$ & ANR & Flavonoid biosynthesis (ko00941) \\
Sobic.006G253900 & 6 & $\mathrm{Up}$ & F3H & Flavonoid biosynthesis (ko00941) \\
Sobic.001G444400 & 1 & Down & POD & Phenylpropanoid biosynthesis (ko00940) \\
Sobic.004G340200 & 4 & Down & CCR & Phenylpropanoid biosynthesis (ko00940) \\
Sobic.006G272700 & 6 & $\mathrm{Up}$ & - & - \\
Sobic.008G026200 & 8 & $\mathrm{Up}$ & - & - \\
Sobic.004G153900 & 4 & $\mathrm{Up}$ & - & - \\
Sobic.001G249600 & 1 & $\mathrm{Up}$ & - & \\
\hline
\end{tabular}

\section{5 qRT-PCR of DEGs Under Salt Treatment}

To illustrate whether the expression of these DEGs is associated with salt tolerance in sweet sorghum, the expression of the 4 up-regulated DEGs identified above was tested by qRT-PCR. In each case, the qRT-PCR data were closely agreement with the transcript patterns that were estimated from the RNA-seq data (Figure 6), which indicated that the RNA-seq data reflected the genes expression model under salt stress. All the 4 genes what had shown an elevated expression in sweet sorghum GZ under salt stress and control based on RNA-sequencing, expressed higher than grain sorghum and were induced expressing by salt treatment based on qRT-PCR, and their responses to salt were faster in GZ than in grain sorghum.

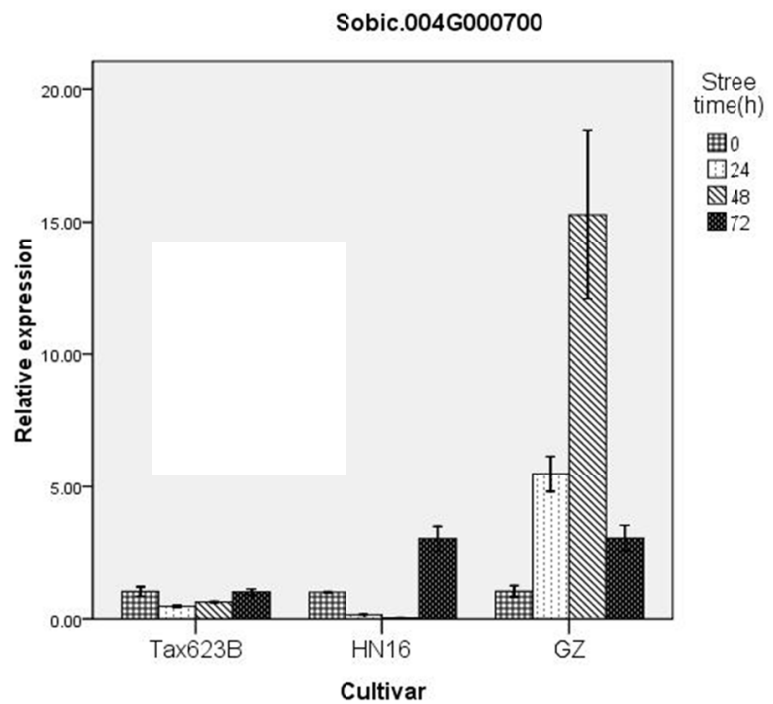

A

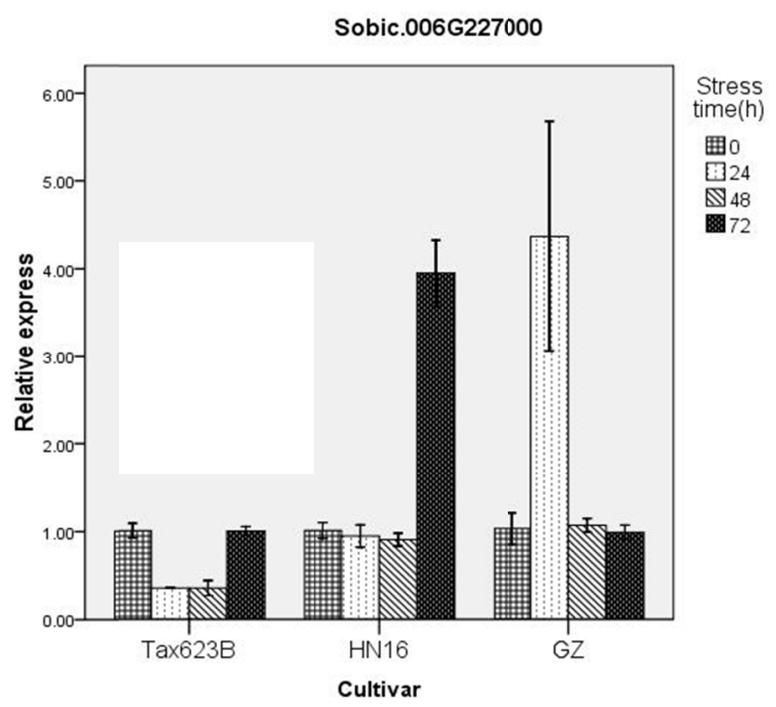

B 


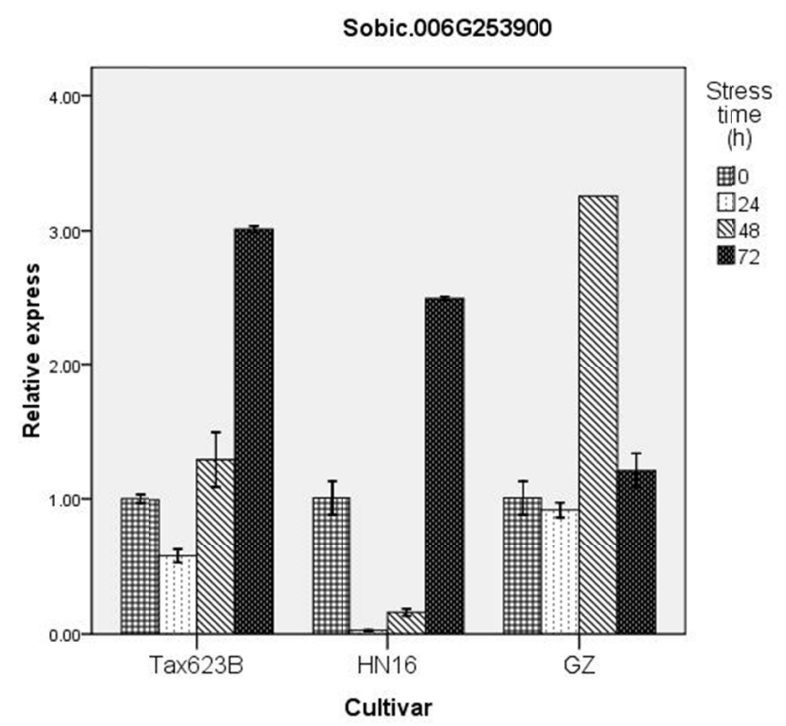

A

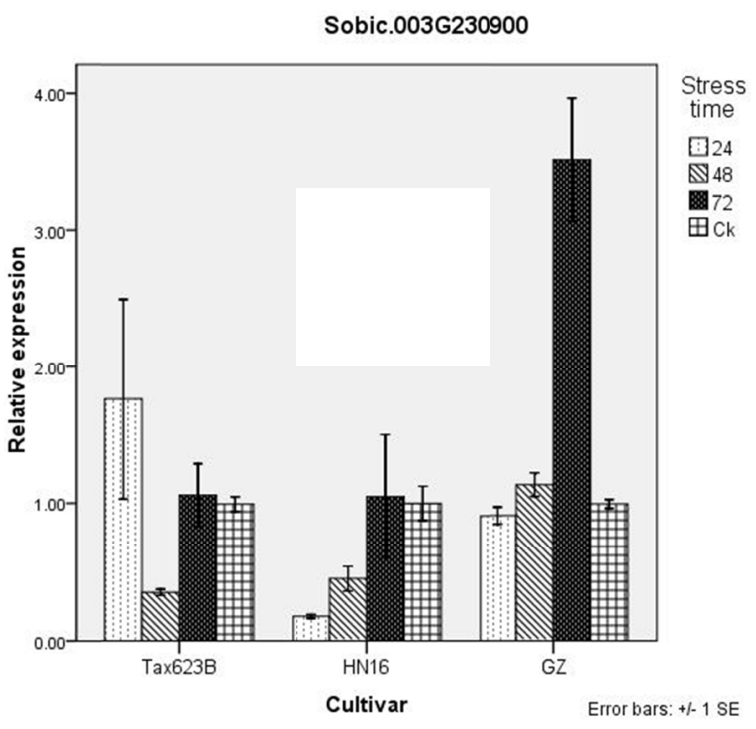

B

Figure 6. qRT-PCR results of 4 DEGs in sweet and grain sorghum. Error bars represent standard errors from three technical replicates

\subsection{Salt Stress Effect on Tannins Accumulation}

Swissprot annotation revealed the 4 up-regulated genes fished out above code DFR, LDOX, ANR and F3H enzymes, which were all involved in the biosynthetic pathway leading to the synthesis of anthocyanins and condensed tannins (proanthocyanidins) (Figure 7; Shimada et al., 2005), and the 2 down regulated genes coding POD and CCR are the key enzymes from phenylalanine to lignin, their down-regulated expressions will help phenylalanine turn to the pathway of synthesis of anthocyanins and condensed tannins (proanthocyanidins). Thus, tannins accumulation at different salt treat time was detected in three sorghum varieties. The results showed tannins content increased after salt stress and was higher in the sweet sorghum than that in grain sorghum (Figure 8). And tannins content changes before and after salt treatment had a positive relationship with the sorghum salt tolerance.

By comparing tannins content at $0,24,48,72 \mathrm{~h}$ after salt stress and the qRT-PCR data, we found that the genes expressions of DFR, LDOX, ANR, and F3H in tannic acid biosynthesis pathway had a positive relationship with tannins content changes before and after salt treatment, and the genes for POD and CCR were negative associated with tannins content changes. These results indicated these genes and flavonoid biosynthesis had an important role in the resistance to salt. 


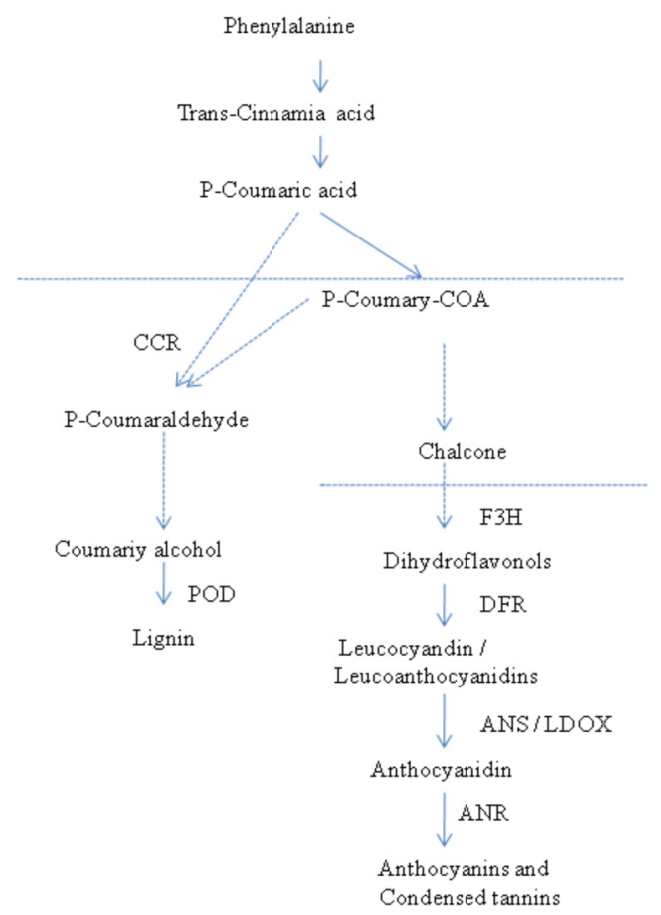

Figure 7. The biosynthetic pathway leading phenylalanine to anthocyanins and lignin

Note. Dotted line represents some steps.

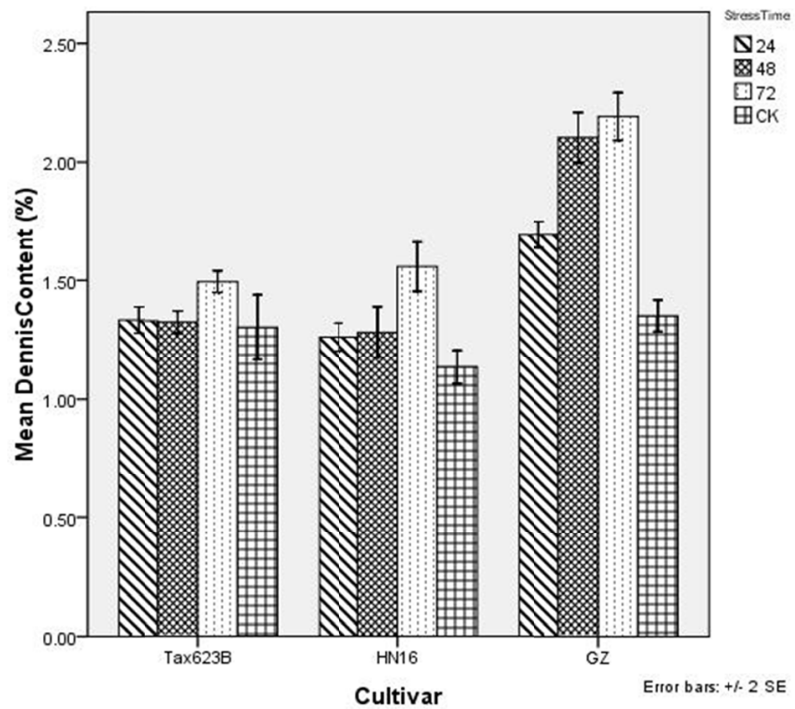

Figure 8. Tannins change in sweet and grain sorghum at different salt stress time, Error bars represent standard errors from three biological replicates

\section{Discussion}

Sorghum is considered to be one of the most abiotic stress tolerant crops, sweet sorghum is one of potential plants for bioenergy production and relatively well adapted to enviromental stress such as water stress, salinity and alkalinity (Maw et al., 2016). Previous studies have shown that some abiotic stress tolerance genes and compounds are resident in sorghum (Singhet al., 2017). Some studies have indicated that secondary metabolism is highly affected by drought-induced oxidative stress. Several metabolites produced by the roots and transported to the shoot are crucial for signal transduction and adaptation to abiotic stress such as water stress (Besseau et al., 2007; Alvarez et al., 2008; Dempsey et al., 2011). In our study, salt stress significantly affected the roots and shoots growth at sorghum seedling stage, and significant differences existed between the sweet and grain 
sorghum in the salt stress inhibition. The analyses based on RNA-sequencing, 6 genes involved in flavonoid biosynthesis were found differential expressed in sweet sorghum GZ compared with grain sorghum. Swissprot annotation revealed the 4 up-regulated genes responsible for DFR, LDOX, ANR and F3H enzymes, were all involved in the biosynthetic pathway leading to the synthesis of anthocyanins and condensed tannins (proanthocyanidins). While, the 2 genes coding POD and CCR which are the key enzymes leading phenylalanine to lignin down regulated, which will help phenylalanine to synthesis of anthocyanins and condensed tannins. The anthocyanin biosynthetic pathway is well understood and is conserved among plants, and its key enzymes and genes have been characterized (Besseau et al., 2007; Hichri et al., 2011.). It is involved in the regulation of the environmental stress responses. Anthocyanin and tannins have high antioxidant capacity and help in the natural defense of plants against abiotic stress damage and pests and diseases (Dempsey et al., 2011).

In this study, the genes responsible for F3H, DFR, LDOX and ANR are key enzymes in anthocyanins and condensed tannins biosynthesis, the 2 genes coding POD and CCR were involved in the pathway from phenylalanine to lignin. Phenylalanine is the pre-material of flavonoid biosynthesis and synthesis of lignin. The changes of these genes expression will affect phenylalanine biosynthesis pathway directions and flavonoids accumulation. The RNA-sequencing data showed F3H, DFR, LDOX and ANR expressed significantly higher in sweet sorghum, while the 2 genes coding POD and CCR expressed lower than that in grain sorghum either under normal or in salt stress conditions. These changes of genes expression would result in the accumulation of anthocyanins or tannins. Meanwhile, the results also showed tannins content changes were positively related with the sorghum salt resistance. It is assumed that accumulation of tannins enhances the sorghum capacity for salt tolerance.

It has been documented that flavonoids have important physiological roles in plants, its accumulation is induced by abiotic stresses and is a hallmark of plant stress (Mori et al., 2005; Meng et al., 2015; Gu et al., 2015). For instance, flavonoid accumulation increases under chilling stress in maize, grape, red orange, apple and tobacco (Meng et al., 2015; Christie et al., 1994). In addition, it was observed that salt-tolerant species often accumulate more flavonoids than the salt-sensitive species, which suggested that there exist a relationship between flavonoids biosynthesis and salt stress resistance (Liu et al., 2012). The results of Meng et al. (2015) study in tobacco demonstrated that both chilling stress and SIF3HL (F3H-like protein gene) overexpression induced flavonoid accumulation in tobacco, implying that SIF3HL plays a key function in flavonoid biosynthesis or in regulating this biosynthetic pathway in response to chilling stress. The increased flavonoid contents may contribute to elevating the antioxidant activity of the plant tissues under stress (Piero et al., 2005; Ubi et al., 2006; López Climen et al., 2008). Our results implied flavonoid biosynthesis pathway played an important role in the high salt tolerance in the landrace sweet sorghum.

In conclusion, salt stress had significant inhibition to sorghum seed germination capability and seeding growth. Six genes involved in flavonoid biosynthesis were found based on RNA-Seq in the landrace sweet sorghum. The analysis results suggested that flavonoid biosynthesis pathway played an important funcion in the sweet sorghum high salt tolerance. This could provide a valuable reference for further understanding of sorghum response to saline stress.

\section{Acknowledgments}

The authors thank Hebei Province Key Basic Research Foundation (15962905D) and Hebei Provincial Department of Land and Resources (2014995161) for funding this research. We thank Dr. Tinashe Zenda for editing help.

\section{References}

Alfenito, M. R., Souer, E., Goodman, C. D., Buell, R., Mol, J., Koes, R., \& Walbot, V. (1998). Functional complementation of anthocyanin sequestration in the vacuole by widely divergent glutathione S-transferases. Plant Cell, 10, 1135-1149. https://doi.org/10.1105/tpc.10.7.1135

Alvarez, S., Marsh, E. L., Schroeder, S. G., \& Schachtman, D. P. (2008). Metabolomic and proteomic changes in the xylem sap of maize under drought. Plant Cell and Environment, 31(3), 325-340. https://doi.org/10.1111/ j.1365-3040.2007.01770.x

Anders, S., \& Huber, W. (2010). Differential expression analysis for sequence count data. Genome Biology, 11, R106. https://doi.org/10.1186/gb-2010-11-10-r106

Besseau, S., Hoffmann, L., Geoffroy, P. C., Pollet, B., \& Legrand, M. (2007). Flavonoid accumulation in arabidopsis repressed in lignin synthesis affects auxin transport and plant growth. Plant Cell, 9(1), 148-162. https://doi.org/10.1105/tpc.106.044495 
Carpita, N. C., \& Mccann, M. C. (2008). Maize and sorghum: Genetic resources for bioenergy grasses. Trends in Plant Science, 13(8), 0-420. https://doi.org/10.1016/j.tplants.2008.06.002

Christie, P. J., Alfenito, M. R., \& Walbot, V. (1994). Impact of low temperature stress on general phenylpropanoid and anthocyanin pathways: Enhancement of transcript abundance and anthocyanin pigmentation in maize seedlings. Planta, 194, 541-549. https://doi.org/10.1007/BF00714468

Deinlein, U., Stephan, A. B., Horie, T., Luo, W., Xu, G., \& Schroeder, J. I. (2014). Plant salt-tolerance mechanisms. Trends in Plant Science, 19(6), 371-379. https://doi.org/10.1016/j.tplants.2014.02.001

Dempsey, D. A., Vlot, A. C., Wildermuth, M. C., \& Klessig, D. F. (2011). Salicylic acid biosynthesis and metabolism. Arabidopsis Book, 9, e0156. https://doi.org/10.1199/tab.0156

Farooq, M., Gogoi, N., Hussain, M., Barthakur, S., Paul, S., Bharadwaj, N, ... Siddique, K. H. M. (2017). Effects, tolerance mechanisms and management of salt stress in grain legumes. Plant Physiology and Biochemistry, 118, 199-217. https://doi.org/10.1016/j.plaphy.2017.06.020

Gu, X. B., Chen, Y. H., Gao, Z. H., Qiao, Y. H., \& Wang, X. Y. (2015). Transcription factors and anthocyanin genes related to low temperature tolerance in rd29A:RdreB1BI transgenic strawberry. Plant Physiology and Biochemistry, 89, 31-43. https://doi.org/10.1016/j.plaphy.2015.02.004

Johnson, D. W., Smith, S. E., \& Dobrenz, A. K. (1992). Genetic and phenotypic relationships in response to $\mathrm{NaCl}$ at different developmental stages in alfalfa. Theoretical and Applied Genetics, 83(6-7), 833-838. https://doi.org/10.1007/BF00226705

Kosová, K., Vítámvás, P., Prášil, I. T., \& Renaut, J. (2011). Plant proteome changes under abiotic stress-Contribution of proteomics studies to understanding plant stress response. Journal of Proteomics, 74(8), 1301-1322. https://doi.org/10.1016/j.jprot.2011.02.006

Liu, C., Li, S., Wang, M., \& Xia, G. (2012). A transcriptomic analysis reveals the nature of salinity tolerance of a wheat introgression line. Plant Molecular. Biollogy, 78(1-2), 159-169. https://oi.org/10.1007/s11103011-9854-1

Livak, K. J., \& Schmittgen, T. D. (2001). Analysis of relative gene expression data using real-time quantitative PCR and the $2^{-\Delta \Delta C T}$ method. Methods, 25(4), 402-408. https://doi.org/10.1006/meth.2001.1262

López, C., María, F., Arbona, M. V., Pérez, C., Rosa, M., \& Gómez, C. A. (2008). Relationship between salt tolerance and photosynthetic machinery performance in citrus. Environmental \& Experimental Botany, 62(2), 176-184. https://doi.org/10.1016/j.envexpbot.2007.08.002

Love, M.I., Huber, W., \& Anders S. (2014). Moderated estimation of fold change and dispersion for RNA-seq data with DESeq2. Genome Biology, 15, 1-34.https://doi.org/10.1186/s13059-014-0550-8

Martin, J. A., \& Wang, Z. (2011). Next-generation transcriptome assembly. Nature Reviews Genetics, 12(10), 671-682. https://doi.org/10.1038/nrg3068

Maw, M. J. W., Houx, J. H., \& Fritschi, F. B. (2016). Sweet sorghum ethanol yield component response to nitrogen fertilization. Industrial Crops and Products, 84, 43-49. https://doi.org/10.1016/j.indcrop.2016. 01.038

Meng, C., Zhang, S., Deng, Y. S., Wang, G. D., \& Kong, F. Y. (2015). Overexpression of a tomato flavanone 3-hydroxylase-like protein gene improves chilling tolerance in tobacco. Plant Physiology and Biochemistry, 96, 388-400. https://doi.org/10.1016/j.plaphy.2015.08.019

Mori, K., Sugaya, S., \& Gemma, H. (2005). Decreased anthocyanin biosynthesis in grape berries grown under elevated night temperature condition. Scientia Horticulturae, 105(3-4), 319-330. https://doi.org/10.1016/ j.scienta.2005.01.032

Munns, R., \& Tester, M. (2008). Mechanisms of Salinity Tolerance. Annual Reviews of Plant Biology, 59, 651-81. https://doi.org/10.1146/annurev.arplant.59.032607.092911

Paterson, A. H., Bowers, J. E., Bruggmann, R., Dubchak, I., Grimwood, J., Gundlach, H., ... Rokhsar, D. S. (2009). The sorghum bicolor genome and the diversification of grasses. Nature, 457(7229), 551-556. https://doi.org/10.1038/nature07723

Piero, A. R. L., Puglisi, I., Rapisarda, P., \& Petrone, G. (2005). Anthocyanins accumulation and related gene expression in red orange fruit induced by low temperature storage. Journal of Agricultural \& Food Chemistry, 53(23), 9083-9088. https://doi.org/10.1021/jf051609s 
Regassa, T. H., \& Wortmann, C. S. (2014). Sweet sorghum as a bioenergy crop: Literature review. Biomass and Bioenergy, 64, 348-355. https://doi.org/10.1016/j.biombioe.2014.03.052

Rooney, T., Bresnihan, B., Andersson, U., Gogarty, M., Kraan, M., Schumacher, H. R., \& Paul, P. T. (2007). Microscopic measurement of inflammation in synovial tissue: Inter-observer agreement for manual quantitative, semiquantitative and computerised digital image analysis. Annals of the Rheumatic Diseases, 66(12), 1656-60. https://doi.org/10.1136/ard.2006.061143

Santos, I., Almeida, A. F., Pirovani, C. P., Costa, M., Silva, M., Bellete, B. S., ... Abelmon, da S. G. (2017). Differential accumulation of flavonoids and phytohormones resulting from the canopy/rootstock interaction of citrus plants subjected to dehydration/rehydration. Plant Physiology \& Biochemistry, 119, 147-158. https://doi.org/10.1016/j.plaphy.2017.08.019

Shimada, N., Sasaki, R., Sato, S., Kaneko, T., Tabata, S., Aoki, T., \& Ayabe, S. (2005). A comprehensive analysis of six dihydroflavonol 4-reductases encoded by a gene cluster of the Lotus japonicus genome. Journal of Experimental Botany, 56(419), 2573-2585. https://doi.org/10.1093/jxb/eri251

Singh, S., Virdi, A. S., Jaswal, R., Chawla, M., Kapoor, S., Mohapatra, S. B., ... Prabhjeet, S. (2017). A temperature-responsive gene in sorghum encodes a glycine-rich protein that interacts with calmodulin. Biochimie, 137, 115-123. https://doi.org/10.1016/j.biochi.2017.03.010

Suja, G., Manoharan, D., Li, J., Monica, B., \& Ajay, P. (2017). Transcriptomic responses to drought and salt stress in desert tree Prosopis juliflora. Plant Gene, 12, 114-122. https://doi.org/10.1016/j.plgene.2017. 09.004

Tripoli, E., Guardia, M. L., Giammanco, S., Majo, D. D., \& Giammanco, M. (2007). Citrus flavonoids: Molecular structure, biological activity and nutritional properties: A review. Food Chemistry, 104(2), 466-479. https://doi.org/10.1016/j.foodchem.2006.11.054

Türkan, I., \& Demiral, R. T. (2009). Recent developments in understanding salinity tolerance. Environmental and Experimental Botany, 67(1), 2-9. https://doi.org/10.1016/j.envexpbot.2009.05.008

Ubi, B. E., Honda, C., Bessho, H., Kondo, S., Wada, M., Kobayashi, S., \& Moriguchi, T. (2006). Expression analysis of anthocyanin biosynthetic genes in apple skin: Effect of UV-B and temperature. Plant Science, 170(3), 571-578. https://doi.org/10.1016/j.plantsci.2005.10.009

Vermerris, W. (2011). Survey of genomics approaches to improve bioenergy traits in maize, sorghum and sugarcane. Journal of Integrative Plant Biology, 53(2), 105-119. https://doi.org/j.1744-7909.2010.01020.x

Wang, W., Muhammad, K. U. R., Feng, J., \& Tao, J. (2017). Rna-seq based transcriptomic analysis of cppu treated grape berries and emission of volatile compounds. Journal of Plant Physiology. https://doi.org/ 10.1016/j.jplph.2017.08.004

Wang, Z. F., Wang, J. F., Bao, Y. M., Wu, Y. Y., Su, X., \& Zhang, H. S. (2010). Inheritance of rice seed germination ability under salt stress. Rice Science, 17(2), 105-110. https://doi.org/10.1016/S1672-6308(08) $60112-2$

Zeng, L., Shannon, M. C., \& Lesch, S. M. (2001). Timing of salinity stress affects rice growth and yield components. Agricultural Water Management, 48(3), 191-206. https://doi.org/10.1016/S0378-3774(00) 00146-3

Zhang, C. X., Bian, M. D., Yu, H., Liu, Q., \& Yang, Z. M. (2011). Identification of alkaline stress-responsive genes of CBL family in sweet sorghum (Sorghum bicolor L.). Plant Physiology and Biochemistry, 49(11), 1306-1312. https://doi.org/10.1016/j.plaphy.2011.08.010

\section{Notes}

Note 1. Download the Supplementary Tables 1 to 6 from https://drive.google.com/open?id=1SPurAdYfQUAnj SO9Bcq5eQBsX5IEWOWD

\section{Copyrights}

Copyright for this article is retained by the author(s), with first publication rights granted to the journal.

This is an open-access article distributed under the terms and conditions of the Creative Commons Attribution license (http://creativecommons.org/licenses/by/4.0/). 